\title{
Urogenitales Menopause-Syndrom mehr beachten
}

\author{
J. Matthias Wenderlein
}

\begin{abstract}
Der Terminus „vulvovaginale Atrophie“ bei postmenopausalen Frauen berücksichtigt zu wenig die Vielfalt an heterogenen Beschwerden, die damit assoziiert sind. Daher wurde 2014 in Chicago von einem internationalen Expertenteam eine Konsensuskonferenz der Nordamerikanischen Menopause-Gesellschaft einberufen. Der neue Terminus lautet nun „Urogenitales Menopausen-Syndrom“. Diese Bezeichnung ist medizinisch umfassender. Größere gesellschaftliche Akzeptanz wird davon erwartet. Immerhin leidet etwa die Hälfte der Frauen ab der Menopause an diesem Syndrom, aber nur wenige bekommen adäquate Therapie so die internationale Expertenkommission.
\end{abstract}

Um für deutsche Frauen in dieser hormonellen Lebensphase therapeutische Verbesserungen zu erreichen, wurde der folgende Beitrag verfasst.

Gefördert und ergänzt werden soll damit klinisches Urteilsvermögen, das den Besonderheiten der Patientensituation gerecht wird. Dies fordert aktuell ein Beitrag zu evidenzbasierter Medizin (Deutsches Ärzteblatt Heft 4 vom 23.1.2015 von O. Leiß C110-112): „Die Qualität der klinischen Betreuung allein an der Befolgung von Leitlinien festmachen zu wollen, ist falsch und wird dem individuellen Patienten nicht gerecht“. Das Urogenitale Atrophie-Syndrom ist dafür ein Paradebeispiel.

\section{Zur Anatomie und Physiologie der Vaginalwand \\ $\nabla$}

Die Vaginalwand ist mit $3 \mathrm{~mm}$ relativ dünn. Die Oberfläche, ein mehrschichtiges, nicht verhornendes Plattenepithel, zeigt nur ganz wenig Präkeratinbildung. Die Oberfläche ist reich an Glykogen und arm an Drüsen, enthält aber reichhaltig elastische Fasern. Dieses Epithel befindet sich in einem ständigen Umbauprozess, der bei Frauen im fertilen Alter abhängig von den Östrogen- und Progesteronkonzentrationen ist. Fallen diese hormonellen Faktoren ab der Menopause weg, so fehlt an der Vaginaloberfläche Glykogen, das als Nährboden für Döderlein-Bakterien dient. Wird mangels Bakterien keine Milchsäure mehr produziert, verändert sich das saure Milieu (pH-Wert: 4-4,5) in Richtung neutral - und damit geht der Schutz vor Infektionen verloren.

\section{Zum klinischen Bild vaginaler Atrophie \\ $\nabla$}

Das Östrogendefizit im Vaginaltrakt zeigt sich bereits in der Vulvaregion mit dünner Haut und abnehmendem Turgor der großen Labien. Die vaginalen Falten verstreichen, die Scheidenhaut erscheint damit „glatt“, trocken, dünner und „durchscheinend“. Damit wird das direkt unter der Oberfläche liegende Venengeflecht sichtbar. Bereits bei der Spekulumeinstellung kann es zu leichten Verletzungen kommen. Kolposkopisch sind oft Petechien erkennbar.

Der vaginale Reifungsindex als lokaler Östrogenstatus ist erniedrigt. Dieser ergibt sich aus dem Anteil von Oberflächenzellen in Relation zu den intermediären und parabasalen Zellen. Insbesondere letztere nehmen unter Östrogenmangel deutlich zu.

\section{Studiendaten aus Deutschland $\nabla$}

In einer Doppelblindstudie [1] wurde mit Scheidenpessaren behandelt, die kontinuierlich 0,2 mg oder 0,03 mg Östriol bzw. ein Placebo abgaben. 436 postmenopausale Frauen mit vaginaler Atrophie wurden erfasst. Diese mussten mindestens ein Symptom haben, das auf einer visuellen Analog-Skala (VAS) (1-100 Punkte) über 65 eingestuft wurde. Dies wurde als deutliche Beschwerdeproblematik bewertet. Erfragt wurden die subjektiv am meisten belastenden Symptome (MBS = most bothersome symptoms). Angestrebt wurden 3 Therapieziele:

1. Der vaginale Maturationsindex (VMI) unter $40 \%$ sollte deutlich angehoben werden.

2. Der Vaginal-pH sollte wieder in den Normbereich (4-4,5) abgesenkt werden.

3. Der MBS sollte von > 65 auf Werte deutlich darunter $(<35)$ abfallen.

All dies sollte innerhalb von 12 Wochen lokaler Therapie erreicht werden. Bei den so selektierten Frauen mit Beschwerden zwischen 65 und 100 Punkten hinsichtlich MBS bzw. auf der VAS-Skala lag die Beschwerde-Intensität der Frauen im Mittel bei 80 Punkten.

Durch die lokale Östrogentherapie wurde im Mittel eine Reduktion um 50 Punkte erreicht, in der Placebogruppe nur um 27 Punkte. Damit lag die Beschwerde-Intensität dieser Frauen oft noch im Bereich um 65 und mehr Punkte, unter Placebo wurde das Therapieziel somit nicht erreicht.

Diese rationale Erfassung des Therapieergebnisses mag eventuell nicht überzeugen. Wurden die Frauen nach 12 Wochen über den Therapieerfolg gefragt, so wurde dieser von $85 \%$ der Patientinnen, die $0,2 \mathrm{mg}$ Östriol vaginal pro Tag erhalten hatten, als sehr gut und gut eingestuft, sowie von $80 \%$ der Frauen, die $0,03 \mathrm{mg}$ Östriol vaginal pro Tag verabreicht bekommen hatten sowie von $45 \%$ Frauen, die Placebo erhalten hatten. 


\section{Östriolnebenwirkungen \\ $\nabla$}

Nebenwirkungen (auch in sehr geringer Form) traten bei $24 \%$ der Patientinnen unter $0,2 \mathrm{mg}$ Östriol, bei $22 \%$ unter $0,03 \mathrm{mg}$ Östriol und bei $26 \%$ der Frauen in der Placebogruppe auf. Am häufigsten wurde mit 7\% Brennen im Vulvabereich angegeben, gefolgt von Schmerz bei der Applikation (3\%) und lokaler Pruritus (2\%). Das traf für alle 3 Gruppen in gleicher Häufigkeit zu. Ernsthafte Nebenwirkung in Form obiger Symptome gab es insgesamt bei 4,6\% der Frauen. Am häufigsten war dabei lokales Brennen mit 1,1\%, aber nur in der Placebogruppe. Wegen Nebenwirkungen kam es in allen 3 Gruppen zu einer Abbrecherquote von $5,7 \%$.

Abschließend zur subjektiven Einschätzung der Therapieverträglichkeit: 95\% unter 0,2 mg Östriol hielten die Therapie für gut verträglich, $89 \%$ unter $0,03 \mathrm{mg}$ Östriol und $80 \%$ unter Placebo.

\section{Sexualmedizinische Aspekte \\ $\nabla$}

Bei der oben referierten Studie waren die 436 Frauen im Mittel 65 Jahre alt ( \pm 8 Jahre). Über die Hälfte der Frauen antwortete bei der Frage nach Dyspareunie nicht, da sie keinen Koitus hatten. Ob der Partner nicht mehr dazu in der Lage war oder die Frauen alleinstehend waren, wurde nicht erfasst. Erinnert sei dabei an die um 7 Jahre höhere Lebenserwartung von Frauen. Bei Partnerbeziehungen mit noch möglichem Koitus dürfte wesentlich häufiger Dyspareunie infolge Atrophie anzunehmen sein.

\section{Einstufung des Phänomens in den USA}

$\nabla$

Nach der Nordamerikanischen Menopause-Gesellschaft machen 40\% postmenopausaler Frauen belastende Erfahrungen mit Urogenital-Atrophie [2]. Aber nur ein Viertel der Frauen mit dieser Östrogenmangelfolge geht zum Arzt, und die hormonelle Therapie ist relativ selten [3].

Das überrascht bei so charakteristischen Symptomen von vaginaler Atrophie wie brennenden Irritationen bei Trockenheitsgefühl im Vaginalbereich. Bei Dyspareunie ist die Folge oft Koitusverzicht mit Belastung der Partnerbeziehung. Aber auch ohne Sexualität kommt es zu Be- schwerden wie Pruritus in der Vulvaregion. All das korreliert mit dem zytologischen Zellbild: Verschiebungen von Superfizial- in Richtung Parabasal- und Intermediärzellen [3].

Erschwerend und die Lebensqualität beeinträchtigend kommen zur VaginalAtrophie in der Regel gestörte Harnblasen- und Urethralfunktionen hinzu. Damit einhergehende Harninkontinenz verunsichert die Frauen so sehr, dass Sexualität zum Tabu wird. Verstärkt wird das durch pathologische Keimbesiedlung im Vaginalbereich und damit übel riechendem Fluor vaginalis - der zusätzliche Beschwerden auslöst.

Wer diesen Circulus vitiosus infolge Östrogenmangels bedenkt - mit deutlich reduzierter Lebensqualität -, möchte den betroffenen Frauen eine kausale Therapie zukommen lassen. Das ist zügig und ohne Risiken mittels lokaler Östrioltherapie seit über 50 Jahren machbar [4-7].

Das gilt es viel häufiger umzusetzen, insbesondere nach Therapie rezidivierender Infektionen im atrophischen Urogenitalbereich. Bei der Prävention erneuter Entzündungen ist selbstverständlich eine Infektion durch den Partner auszuschließen.

\section{Warum ist die Östrioltherapie gefahrlos? \\ $\nabla$}

Diese Frage lässt sich biologisch relativ einfach beantworten: Östriol ist ein Kurzzeit-Östrogen mit nur kurzer Bindungszeit an den Zellkern [8]. Damit kommt es nicht zur Zellproliferation am Endometrium - als sensibler Indikator für solche Zellveränderungen [9]. Da dies sogar für Östrioldosen von $0,5-1 \mathrm{mg}$ täglich gilt, können nun verwendete lokale Dosierungen von 0,2 und $0,03 \mathrm{mg}$ als risikolos eingestuft werden [10]. Das ist besonders relevant für Frauen nach Brustkrebsbehandlung.

Überraschenderweise reichen so niedrige lokale Östrogendosierungen für Proliferationseffekte an Epithelien in Scheide, Urethra und Harnblase aus. Das führt zugleich zu besserer Durchblutung und damit besserer Funktionalität dieser Gewebe bzw. der Organe insgesamt. Sogar Inkontinenzprobleme infolge Östrogenmangel, die erstmals ab der Menopause auftraten, werden so reduziert bzw. beseitigt. Extreme Lageanomalien von Urethra und Harn- blase schon vor der Menopause, z.B. durch Geburten, lassen solche Erfolge natürlich nicht erwarten.

In der eingangs zitierten Studie ließ sich der vaginale Reifungsindex bereits nach 20 Tagen durch so geringe Östrioldosen erreichen, z.B. Anstieg von 10 auf $60 \%$. Wenn nach 3 Wochen keine Verbesserungen eintreten, dann ist nach anderen Ursachen zu fahnden. Erst dann ist eine höhere lokale Östrioldosierung zu erwägen.

\section{Wie kommt ein Erfolg durch so niedrige Dosierungen zustande? $\nabla$}

Östriol gelangt problemlos auch durch atrophisches Oberflächenepithel hindurch. Dann erst wird das so diffundierte Östriol verstoffwechselt, also in den tieferen Epithelschichten wirksam und „verbraucht". Damit kommt keine nennenswerte systemische Wirkung zustande. Die Dosierung von 0,03 mg Östriol ist seit Oktober 2013 zugelassen, also bereits über 1,5 Jahre und ist GKV-erstattungsfähig.

Für wissenschaftliche Studien sind Bestimmungen des vaginalen Reifungsindex (VMI) und des Vaginal-pH-Wertes angezeigt. Bei Indikationen zu dieser lokalen Östrogentherapie reicht in der Regel die Anamneseerhebung samt klinischem Befund. Beides ist gut zu dokumentieren. Will man die Ausgangssituation unter „semiquantitativen“ Aspekten festhalten, so reicht es, den Therapieerfolg nach Schulnoten von 1-6 ( 1 = sehr gut und $6=$ keinerlei Besserung) beurteilen zu lassen.

Bei der niedrigen lokalen Dosierung von 0,03 mg kommt es nachweislich zu keiner Östriolakkumulation. Die systemische Bioverfügbarkeit ist zu niedrig, um am Endometrium wirksam zu werden. Daher sind keine sonografischen Kontrollen notwendig. Für Brustzellen ist Analoges anzunehmen - wegen der nur kurzen Bindungszeit an Zellkernen bzw. an Hormonrezeptoren.

Die lokale Östriolwirkung setzt prompt ein und zeigt sich bereits 12 Stunden nach Applikation. Nach weiteren 12 Stunden sinken die Östriolserumwerte unter die Nachweisgrenze von $5 \mathrm{pg} / \mathrm{ml}$ ab.

Wiederholt sei, dass der zelluläre Östriolmetabolismus in den Zellen der Vaginalhaut so zügig „vor Ort“ abläuft, dass es 
nicht zu nennenswerten systemischen Absorptionsvorgängen für Östriol kommen kann - das ist auch den Patientinnen mitzuteilen.

In einer Metaanalyse aus 12 Studien mit mindestens 2-jähriger Östrioltherapie [11] wurden sonografische Befunde und Histologien ausgewertet: Das Ergebnis war beruhigend; es fanden sich keinerlei Hinweise auf Endometriumproliferation.

\section{Essigsäuretest nur gezielt einsetzen $\nabla$}

Dieser Test mit 3-5\%iger Essigsäure ist unspezifisch und wenig sensitiv. Kamen früher Frauen nach der Menopause mit Schmerz, Brennen und Jucken in der Vulvaregion in die gynäkologische Ambulanz - vom Autor zu Beginn der Weiterbildungszeit erlebt -, so wurde „reflexartig“ mit Essigsäure betupft. Die damit sichtbaren weißen Stellen wurden zur histologischen Befundung großzügig biopsiert. In der Regel folgte ein unauffälliger Befund.

Bei Vulvodynie ist erst die Vulvoskopie durchzuführen - ohne Schmerzen. Zeigt sich eine Atrophie, so reicht das für die Indikation lokal niedrig dosierter Östrioltherapie à $0,03 \mathrm{mg}$ täglich. Nach 3 Wochen erfolgt erneut eine klinische Untersuchung samt Vulvoskopie. Erst jetzt sollte bei Auffälligkeiten gezielt die Essigsäure-Probe durchgeführt und $\mathrm{PE} /$ Stanze für eine Histologiebefundung vorgenommen werden.

Vulväre intraepitheliale Neoplasien (VIN) als prämaligne Erkrankungen haben sich hinsichtlich der Inzidenz in den letzten Dezennien verdoppelt. Das mittlere Erkrankungsalter sank von über 60 Jahren auf unter 40 Jahre. Mehr HPV-Infektionen sind eine Ursache. Inzidenzen von 7 von 100000 Frauen werden angegeben - je nach Diagnostikverfahren schwankend. Hier interessiert nicht die Dysplasie-Klassifizierung nach VIN I, II, III.

Die Entwicklung von Vulvakarzinomen lässt sich nicht ausschließlich auf HPV zurückführen. Dazu bedarf es Kofaktoren wie Nikotinkonsum und genetischer Disposition. Stets ist zu bedenken, dass es sich bei Vulvakarzinomen bei Frauen ab der Menopause zu 90\% um Plattenepithelkrebs handelt. Daher kann die lokale Östriolbehandlung innerhalb weniger
Wochen keine Befundverschlechterung provozieren, da Plattenepithelpathologien hormonunabhängig entstehen. Vulvakrebs macht ca. 5\% der Genitalkrebse aus mit einer Erkrankungsrate von 2 pro $100000 /$ Jahr. Das entspricht etwa 35004000 Neuerkrankungen pro Jahr. Das trifft wohl vor allem Frauen ab 60 Jahren und trotzdem ist bei vulvovaginalen $\mathrm{Be}$ schwerden erst an Hormonmangel $\mathrm{zu}$ denken.

\section{Vaginalmykosen vorher behandeln \\ $\nabla$}

Besteht bei Vaginal-Atrophie der Verdacht auf eine Pilzinfektion, dann wird bei Bestätigung sofort mit lokaler Clotrimazoltherapie à $500 \mathrm{mg}$ begonnen. Bei dessen breitem Spektrum kann ein einziges Ovulum genügen, da dieses über 3 Tage genügend Wirkstoff abgibt. Damit werden die Symptome Juckreiz, Rötung, Brennen und vermehrt Fluor gestoppt. Milchsäure-Formulierungen gibt es auf dem Markt. Wichtig ist es aber, anschließend eine lokale Östrioltherapie zur Rezidivprophylaxe vorzunehmen.

Zusätzliche Vitamin-D-Gaben gegen Rezidive bei bakteriellen Vaginosen haben sich als unwirksam erwiesen bzw. stellen nur eine „Laborkosmetik“ dar.

\section{Lokale Östrioltherapie nach Brustkrebs vertretbar? $\nabla$}

Frauen mit Brustkrebs belastet die Therapie mit Aromatasehemmern wegen Vaginalatrophie erheblich. Die Furcht vor kausaler Therapie mit Östriol konnte eine Studie an 16 Frauen widerlegen [11].

Mit umfangreicher Labordiagnostik ließ sich nachweisen, dass Östriol 0,03 mg lokal für 4 Wochen täglich und danach 8 Wochen lang 3-mal wöchentlich nur sehr kurzfristig zum Östriolanstieg im Serum führte. Dagegen wurden Östron und Östradiol nicht erhöht.

Da schon so geringe lokale Östrioldosierungen den Frauen deutliche Beschwerdebesserung bringt und damit bessere Lebensqualität, sollte dies häufiger genutzt werden. Bestätigend sei dazu eine Fallkontrollstudie angeführt [12], die nachwies, dass durch vaginale Östrioltherapie das Rezidivrisiko bei Frauen mit Brustkrebs nicht erhöht wurde. Das bestätigte eine weitere Studie [13] sowie eine retrospektive Kohortenstudie aus Finnland [14].

Warum trotzdem die onkologische Angst vor Östriol besteht, ist schwer nachvollziehbar. Denn Östriol stellt das Endprodukt der Östrogenmetabolisierung dar. Es kann also nicht in Östron und/oder Östradiol zurückverwandelt werden $[15,16]$. Nur vor letzterem könnte onkologisch Furcht bestehen.

Nochmals sei wiederholt, dass Östriol eine um den Faktor 10 geringere Affinität zum Östrogenrezeptor hat als Östradiol [16]. Die Bindungszeit ist weniger als halb so lang unter Östriol und zwar nur 6 Stunden gegenüber 12 Stunden unter Östradiol [17].

\section{Aromatasehemmer induzieren sexuelle Dysfunktion bei 9 von 10 Frauen \\ $\nabla$}

Texanische Onkologen [18] erfassten mit standardisiertem Erhebungsbogen sexuelle Dysfunktion unter Aromatasehemmern, die seit 1,5-2 Jahren eingenommen wurden. Die Frauen waren zu Therapiebeginn durchschnittlich 63 Jahre alt und hatten zur Hälfte sexuelle Aktivität angegeben. Bei 93\% wurde unter Aromatasehemmertherapie eine Dysfunktion festgestellt. Das erlebten 3 von 4 Frauen als sehr belastend. Die Hälfte versuchte dem mit Vaginalcremes ohne Hormone abzuhelfen, und ein Viertel entschied sich zur sexuellen Enthaltsamkeit.

Lokale Östrogentherapie war in dieser texanischen Studie kein Thema. Das überrascht, da die Aromatasehemmertherapie mit adjuvanter Indikation erfolgte. Damit bekamen sicher viele Frauen in frühem Brustkrebsstadium eine Behandlung.

\section{Diskussion um „Östrogen als Brustkrebsrisiko“ beenden $\nabla$}

In großer Mehrheit werden Brustkrebsdiagnosen erst nach der Menopause gestellt, also in der Hypogonadismusphase der Frau. In der Women's Health Initiative gab es unter konjugiertem Östrogen im Vergleich mit Placebo signifikant weniger Brustkrebsdiagnosen. Das wurde auch in Nachbeobachtungen bestätigt und gilt auch für Deutschland: nach GKV-Daten gibt es bei Nutzerinnen von Hormonsub- 
stitution ein Drittel weniger Brustkrebsdiagnosen [19]. Noch beeindruckender sind mehrere Studien, die bei Frauen nach Brustkrebsbehandlung und späterer Östrogensubstitution einen onkologischen Benefit feststellten [20]. Fazit daraus: Östriol lokal in heutigen niedrigen Dosierungen ist ohne Krebsrisiko.

\section{Zusammenfassung}

Lokale Östrioltherapie in nun geringer Dosierung von $0,03 \mathrm{mg}$, im Gegensatz zu vorher mit $0,5 \mathrm{mg}$ - d.h. um Faktor 17 niedriger, ist möglich. Damit sind in der Regel klinische Erfolge erzielbar.

Wegen kurzen Bindungszeiten mit deutlich geringerer Rezeptor-/Zellkern-Affinität im Vergleich zu Östradiol, ist Östriol lokal ohne onkologische Risiken. Systemische Wirkungen von klinischer Relevanz ließen sich - via Östriolbestimmungen im Serum und histologische Aufarbeitung von Endometrium auch nach längerer lokaler Östriolanwendung - ausschließen.

\section{Literatur}

1 Griesser H, Skonietzki S, Fischer T et al. Low dose estriol pessaries for the treatment of vaginal atrophy: a double-blind placebocontrolled trial investigating the efficacy of pessaries containing $0.2 \mathrm{mg}$ and $0.03 \mathrm{mg}$ estriol. Maturitas 2012; 71: 360-368

2 The North American Menopause Society. The role of local vaginal estrogen for treatment of vaginal atrophy in postmenopausal women: 2007 position statement of the North American Menopause Society. Menopause 2007; 14: 355-363
3 Nappi RE, Kokot-Kierepa M. Women's voices in the menopause: results from an international survey on vaginal atrophy. Maturitas 2010; 67: 233-238

4 Puck A, Korte W, Hübner KA. Die Wirkung des Oestriol auf Corpus uteri, Cervix uteri und Vagina der Frau. Dtsch Med Wschr 1957; 82: 1864-1866

5 Gitsch E, Golob E. Zur Frage der idealen Oestriolkonzentration in Salben bei genitaler Anwendung. Zbl Gynaekol 1962; 84: 454-458

6 Suckling J, Lethaby A, Kennedy R. Local estrogen for vaginal atrophy in postmenopausal women. Cochrane Database Syst Rev 2006; 4: CD001500

7 North American Menopause Society. Estrogen and progestogen use in postmenopausal women: 2010 position statement of The North American Menopause Society. Menopause 2010; 17: 242-255

8 Bergink EW. Oestriol receptor interactions: their biological importance and therapeutic implications. Acta Endocrinol (Copenh) 1980; 233: 9-16

9 Vooijs GP, Geurts TB. Review of the endometrial safety during intravaginal treatment with estriol. Eur J Obstet Gynecol Reprod Biol 1995; 62: 101-106

10 Buhling KJ, Eydeler U, Borregaard S et al. Systemic bioavailability of estriol following single and repeated vaginal administration of $0.03 \mathrm{mg}$ estriol containing pessaries of vaginal atrophy. Arzneimittelforschung 2012; 62: 378-383

11 Dondres G, Neven P, Moegele $M$ et al. CLINICAL TRIAL Ultra-low-dose estriol and Lactobacillus acidophilus vaginal tablets (Gynoflor $^{\circledR}$ ) for vaginal atrophy in postmenopausal breast cancer patients on aromatase inhibitors: pharmacokinetic, safety, and efficacy phase I clinical study. Breast Cancer Res Treat 2014; 145: 371-379

12 O'Meara ES, Rossing MA, Daling JR et al. Hormone replacement therapy after a diagnosis of breast cancer in relation to recurrence and mortality. J Natl Cancer Inst 2001; 93: 754-762
13 Dew JE, Wren BG, Eden JA. A cohort study of topical vaginal estrogen therapy in women previously treated for breast cancer. Climacteric 2003; 6: 45-52

14 Lyytinen H, Pukkala E, Ylikorkala O. Breast cancer risk in postmenopausal women using estrogen-only therapy. Obstet Gynecol 2006; 108: 1354-1360

15 Head KA. Estriol: safety and efficacy. Altern Med Rev 1998; 3: 101-113

16 van der Vies J. The pharmacology of oestriol. Maturitas 1998; 4: 291-299

17 Anderson JN, Peck EJ jr., Clark JH. Estrogeninduced uterine responses and growth: relationship to receptor estrogen binding by uterine nuclei. Endocrinology 1975; 96: 160-167

18 Schover LR, Baum GP, Fuson LA et al. Sexual problems during the first two years of adjuvant treatment with aromatase inhibitors. J Sex Med 2014; 11: 3102-3111

19 Wenderlein JM. Ein Drittel weniger Brustkrebs-Diagnosen unter HRT. Frauenarzt 2011; 52: 122-123

20 Wenderlein JM. Hormonsubstitution nach Brustkrebs. ÄP Gynäkologie 2014; 6: 10-11

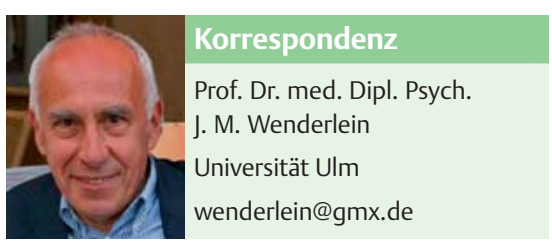

